\title{
The Motor Kinesin 4II Is Important for Growth and Chloroplast Light Avoidance in the Moss Physcomitrella patens
}

\author{
Daniel MacVeigh-Fierro', Erkan Tüzel ${ }^{2}$, Luis Vidali1 ${ }^{*}$ \\ ${ }^{1}$ Department of Biology and Biotechnology, Worcester Polytechnic Institute, Worcester, MA, USA \\ ${ }^{2}$ Department of Physics, Worcester Polytechnic Institute, Worcester, MA, USA \\ Email: *lvidali@wpi.edu
}

How to cite this paper: MacVeigh-Fierro, D., Tüzel, E. and Vidali, L. (2017) The Motor Kinesin 4II Is Important for Growth and Chloroplast Light Avoidance in the Moss Physcomitrella patens. American Journal of Plant Sciences, 8, 791-809.

https://doi.org/10.4236/ajps.2017.84055

Received: January 21, 2017

Accepted: March 26, 2017

Published: March 30, 2017

Copyright $\odot 2017$ by authors and Scientific Research Publishing Inc. This work is licensed under the Creative Commons Attribution International License (CC BY 4.0).

http://creativecommons.org/licenses/by/4.0/

\begin{abstract}
Understanding how plant cells adapt dynamically to changes in the environment is a fundamental problem of plant biology. Under many conditions, plant cells respond to environmental changes by modifying their intracellular organization. A critical example of intracellular reorganization is chloroplast photo-relocation, which is required for optimal energy harvesting and avoiding photodamage. A key system responsible for the spatial organization of intracellular components is the microtubule cytoskeleton and its associated motor proteins, kinesins. Here we tested the hypothesis that members of the kinesin 4II subfamily are important for chloroplast photo-relocation in the moss Physcomitrella patens. Most land plants, including $P$. patens, use an actin cytoskeleton-dependent mechanism to transport chloroplasts in response to light. In addition to the actin-based system, $P$. patens can also transport chloroplasts via a microtubule-dependent mechanism, which is absent in flowering plants. Here, we used a $P$. patens line that contains an inducible RNAi system to silence all three kinesin 4-II genes present in this moss and evaluated their participation in the microtubule-dependent chloroplast light avoidance response. Because we found a significant effect on cell growth when kinesin 4IIs are silenced, we took advantage of the inducible system to establish a reproducible and quantitative assay to evaluate chloroplast photo-relocation in full-grown cells. Using a laser scanning confocal-based chloroplast light avoidance response assay, we found a reduction in chloroplast motility when kinesin 4IIs were silenced. Hence, in addition to identifying a role for kinesin 4II proteins in protonemal cell growth, our results strongly support the hypothesis that these kinesins play an important role in the chloroplast light avoidance response.
\end{abstract}

\section{Keywords}

Microtubules, Cytoskeleton, Chloroplast Motility, Polarization, Photo-Relocation 


\section{Introduction}

Chloroplasts are organelles that can be found in most plant and algal cells. They are essential for photosynthesis and therefore the survival of plant and algae species. In these cells, chloroplasts have the ability to move to different locations within the cell depending on the light intensity they are exposed to [1] [2] [3]. In conditions of low light intensity, chloroplasts tend to congregate to the light source in order to gather the most light, thereby increasing photosynthetic activity [4]. On the other hand, when faced with conditions of high intensity light, the chloroplasts avert the light source in order to minimize photodamage [4] [5]. In most plant species, these avoidance and accumulation responses are driven by blue light, but in the moss Physcomitrella patens, chloroplasts have been shown to also respond to red light [6] [7] [8]. The blue light responses are mediated by the light receptors, phototropins, which control plant phototropism [9] and the chloroplast responses to light [7] [10] [11]. How phototropins regulate chloroplast motility in vascular and nonvascular plants is not well understood. What is clearly established is that, downstream from the light signal, in all vascular plants the actin cytoskeleton plays an important role in chloroplast movement [12] [13], while in $P$. patens the microtubule cytoskeleton also participates in chloroplast transport [8] [14].

The moss $P$. patens has been extensively used to investigate many aspects of plant cell biological research. Several reasons, important for biological studies, make this moss a valuable model organism. First, its whole genome has been sequenced, is easily accessible, and is well-annotated [15] [16]. In addition, the moss is in a haploid stage for most of its life cycle, this makes manipulating the DNA of moss easy and its amenable to rapid genetic screening [15] [17]. One of the predominant cell types of this haploid stage is chains of filamentous cells called protonemata, which are amenable to a variety of observations under the microscope. $P$. patens can also undergo high-efficiency mitotic homologous recombination [18] [19] in addition to rapid RNA interference (RNAi) [20] [21]. These techniques are valuable because they allow the manipulating of the DNA or RNA of certain sequences in the moss to knock out or knock down the expression of certain genes to determine their function.

Because they are easily accessible, the cells of moss cells have been extensively used to characterize chloroplast motility. The chloroplast response to blue light was initially identified in Funaria hygrometrica and Ceratodon purpureus [22] [23], but a red light response was not detected in these species. It was subsequently showed in $P$. patens that chloroplasts can respond to red light, but that this response was masked under white light-culturing and required red light-culturing to be detected [6]. In $P$. patens, the blue light accumulation and avoidance responses are driven by either actin or microtubule-dependent mechanisms, but the red responses are mediated only by microtubules [8]. This double system of motility differs with vascular plants were only an actin-dependent system is present [12]. The actin-dependent pathway, composed of short actin filaments on chloroplasts (cp-actin), was found to also be present in P. patens [13]. Furthermore, critical 
components of this conserved system, including the kinesin-like protein KAC, and the chloroplast membrane-associated actin-biding protein CHUP1 have also been shown to be important for actin-based chloroplast photo-relocation in $P$. patens [14] [24] [25].

Plants are similar to other eukaryotes in that the actin and microtubule cytoskeletons act as tracks for the motor proteins myosin and kinesins, respectively. Nevertheless, the actin-based chloroplast photo-relocation mechanism is likely myosin independent [26]. On the other hand, the microtubule-dependent mechanism present in P. patens may depend on kinesins. In plants, as in other eukaryotic organisms, kinesins belong to a large superfamily of molecules with many members conserved in evolution [27]. Microtubules provide tracks for kinesin-based transport, but kinesins are also important for mitosis and cytokinesis; they participate in aligning chromosomes and separating them during mitosis [28], and in plants they also participate in the organization of the phragmoplast, which mediates cytokinesis [29] [30] [31]. In the genome of $P$. patens there are 71 kinesin genes, which can be classified into at least 14 different families [32]. Many of these kinesins were shown to be associated with the spindle or phragmoplast by experiments involving a fluorescent protein tag to their C-terminus [33]. Nevertheless, the research of Miki et al. (2014) failed to identify kinesins associated with chloroplasts in moss. This could have been the result of high chloroplast background, or that expression levels were below their detection methods.

Because of the microtubule dependent transport of chloroplasts in P. patens [8], it is expected that a subset of kinesins may mediate this transport. One intriguing possibility for a candidate motor was offered by the kinesin-like protein, KAC, initially identified in Arabidopsis thaliana and shown to be important for actin-dependent chloroplast motility [34]. In fact, this protein was also found to be important for chloroplast motility in $P$. patens [25], but similarly to vascular plants KACs were found to act via an actin-dependent mechanism [14]. The actin-dependent function of a kinesin-like molecule suggests a time in plant evolution were the two cytoskeletal systems interacted more intimately for chloroplast motility; but this mechanism seems to pre-date the time of divergence between bryophytes and vascular plants.

Searching for additional kinesin molecules that may be responsible for chloroplast motility, we focused on the phylogenetic analysis of Shen et al. (2012), which shows kinesin 4IIs as a monophyletic group composed of three members with no orthologues in $A$. thaliana [32]. Furthermore, the subfamily of kinesins 4II did not seem to localize to the spindle or the phragmoplast [33], suggesting a possible participation in chloroplast motility. In other systems, kinesin $4 \mathrm{~s}$ have been implicated in organelle and chromosome transport [35]. In vascular plants, kinesin 4I proteins have been characterized because mutants of these genes result in small and fragile plants [36] [37]. These kinesins are highly processive motors that transport intracellular cargo important for cell wall formation [38] [39] [40]. In $P$. patens, one kinesin 4I isoform was localized to chromosomes, 
spindle midzone, and phragmoplast's equator [33], where they may function similarly to their animal counterparts in regulating microtubule length during cell division [41]. The functions of kinesin 4II proteins have not been investigated, but due to their conserved motor domain, they are likely to share some properties with the kinesin $4 \mathrm{I}$ molecules, such as plus end directed motility and high processivity [38]. Although the motor domains of the two classes of kinesin 4 molecules are similar, their C-termini are more divergent and shorter in the kinesin 4II proteins; this is likely the result of association with different cargo. The phylogenetic evidence described above suggests that kinesin 4IIs may belong to a subfamily important for microtubule-dependent chloroplast transport, absent in other plants, but present in $P$. patens.

To investigate the participation of kinesin 4IIs in chloroplast motility, here we used an inducible RNAi system. In this system, the expression of the silencing construct remains off until the cell is exposed to the inducer estradiol [21] [42]. We took advantage of the collection of inducible kinesin-RNAi lines generated previously in P. patens by Miki and coworkers [43] to test the participation of kinesin 4IIs in chloroplast motility. Here, we show that silencing kinesin 4II results in a loss of growth phenotype, and in addition, that this kinesin subfamily is important for the chloroplast photo-avoidance response.

\section{Materials and Methods}

\subsection{Phylogenetic Analysis}

To identify representative protein sequences for plant kinesin $4 \mathrm{~s}$ we searched the Phytozome v12 repository (phytozome.jgi.doe.gov). To search, we used the amino acid sequence of the motor domain of kinesin 4IIa from Physcomitrella patens (first 362 amino acids of protein Pp3c13_15600V3.1). Using the BLAST algorithm against the proteomes of 14 selected species we identified 947 protein hits. We selected the following species for analysis: all available Chlorophyte algae (Chlamydomonas reinhardtii v5.5, Dunaliella salina v1.0, Volvox carteri v2.1, Coccomyxa subellipsoidea C-169, v2.0, Micromonas pusilla CCMP1545 v3.0, Micromonas sp. RCC299 v3.0, Ostreococcus lucimarinus v2.0), all available Bryophytes (Marchantia polymorpha v3.1, Physcomitrella patens v3.3, Sphagnum fallax v0.5), a single available Lycopodiophyta species (Selaginella moellendorffii v1.0), three representative well-annotated angiosperms (Ananas comosusv3, Oryza sativa v7, Arabidopsis thaliana TAIR10).

To enrich for members of the kinesin 4 family, we identified the position the kinesin 2 member from $P$. patens in the BLAST results list and selected all sequences with a smaller $\mathrm{E}$ value than $1.5 \times 10^{-80}$. This resulted in a total of $87 \mathrm{se-}$ quences. From this list, we selected only the proteins derived from the primary transcripts, resulting in 53 sequences. Following a simple analysis with ClustalW alignment and neighbor-joining tree build method in Geneious R7, we removed all kinesin $5 \mathrm{~s}$ and kinesin $2 \mathrm{~s}$ with the exception of $P$. patens kinesin 2, which was used for rooting the final tree (see below). This resulted in 39 sequences. To identify the motor domain regions, we aligned the 39 sequences together with 
the head domain of human kinesin 1. The homologous regions were selected from all sequences, and the amino acid positions are listed in Supplementary Table 1. We found kinesin 4 sequences in all species analyzed except in the algae Dunaliella salina and Ostreococcus lucimarinus. This may be the result of incomplete annotations or a true absence in their genome; we did not pursue further analysis for these species.

The motor domains of all the selected kinesin 4s were aligned in Geneious R7 using the ClustalW algorithm with default settings. Because the homology is high between kinesin motor domains, it was not necessary to manually adjust the resulted alignment. To generate the final phylogenetic tree, we used the Maximum-Likelihood algorithm (PhyML) available as a plugin for Geneious R7 [44] with default settings, except a number of bootstraps set to 1000 and no optimization. A consensus tree was subsequently generated where any branch support with a bootstrap value under $50 \%$ was considered not significant. The tree was rooted using the kinesin 2 sequence from $P$. patens and the tree was displayed with Geneious R7 indicating the percent bootstrap support for each branch.

\subsection{Cell Lines and Culture Conditions}

All cell lines used in this study were derived from the moss Physcomitrella patens (Hedw.) Bruch \& Schimp., Gransden strain. Plants were subcultured by grinding weekly on $\mathrm{PpNO} 3$ or $\mathrm{PpNH} 4$ pates made from commercial packets (Caisson Laboratories Inc., Utah), $7 \mathrm{~g}$ of plant agar and $1 \mathrm{~L}$ of $\mathrm{dH}_{2} \mathrm{O}$. For the RNAi lines the culturing process was the same with only a couple differences. Initially, to make a plate where the kinesin would be knockdown for imaging, the RNAi line would be plated on normal $\mathrm{PpNO}_{3}$ plates for four to seven days, then switched the cellophane holding the moss to a $\mathrm{PpNO}_{3}$ plate with $1 \mu \mathrm{M}$ estradiol for three days. This would allow for the RNAi system to be activated preventing new kinesins of the line in question to be made and any kinesin that were left over to degrade. The $\mathrm{PpNO}_{3}$ with $1 \mu \mathrm{M}$ estradiol plates were prepared from a $100 \mathrm{mM}$ estradiol stock in water. For our experiments we used a $P$. patens cell line kindly provided by Dr. Goshima (Division of Biological Science, Graduate School of Science, Nagoya University, Japan) where an inducible construct that silences the kinesin 4II subfamily is present [43]. The line selected for most of the work was RNAi for kinesin 4IIb 359-1. For controls, we used the parental line originally used to make the kinesin 4II-RNAi line as well as the inducible line in the absence of estradiol.

\subsection{Growth Assay}

Protonemal growth was evaluated from regenerating protoplasts using previously published conditions [45] [46]. In order to test the effects of knocking down kinesin $4 \mathrm{II}$ the following modifications were made: Protoplast were regenerated for 4 days with no estradiol, then moved to plates with $1 \mu \mathrm{M}$ estradiol for 4 days while controls were not treated with estradiol. Cells were transferred 
to agar pads and stained with $10 \mu \mathrm{g} / \mathrm{ml}$ calcoflour and imaged using fluorescence microscopy. Area and solidity values per plant were measured using an ImageJ macro as previously described [46].

\subsection{Chloroplast Light Avoidance Response Assay}

Slides containing live moss were prepared for microscope viewing by mounting the cells on a thin layer of $200 \mu \mathrm{l}$ of $\mathrm{PpNO}_{3}$ agar containing $25 \mu \mathrm{M}$ latrunculin B (diluted form a $5 \mathrm{mM}$ stock in ethanol). This agar was placed on a slide with parallel strips of tape on either side of the agar to from a pad and subsequently removed. Small squares of cellophane from the desired plate of moss were cut and placed face down on the solidified agar, left to set for $1-2 \mathrm{mi}-$ nutes before the cellophane was removed carefully with tweezers. Liquid medium $(20 \mu \mathrm{l})$ containing $25 \mu \mathrm{M}$ latrunculin $\mathrm{B}$, to depolymerize actin filaments, was added to the agar pad before covering with a coverslip. The coverslip was then sealed to the slide using melted vaseline, lanolin, and paraffin combined in equal parts.

Cells were analyzed using a laser scanning confocal system (Leica-SP5) using a 40X oil lens (NA 1.2). Moss cells that were being observed were always sub-apical, and the sub-apical cell also must have had enough room for the chloroplasts to move as necessary. Once a specific cell was chosen it was zoomed to a value of three and the laser rotated until the middle of the cell was horizontal on the screen, the confocal pinhole was set to $1 \mathrm{AU}$. The format was set to $512 \times 185$ pixels along with a $30 \times 30 \mu \mathrm{m}$ ROI is set up in the center of the cell for blue light illumination. Only this ROI portion of the cell was exposed to 488 $\mathrm{nm}$ blue light laser at $10 \%$ for 20 minutes, this initiates an avoidance response in the chloroplasts. The rest of the cell was illuminated with a $591 \mathrm{~nm}$ laser at $5 \%$, which illuminates the rest of the cell without causing any chloroplast response. A double dichroic 488/561 nm mirror separates the emission for the excitation laser the emission is then captured by two different detectors. One detector (PMT4) was set at $\sim 700 \mathrm{~nm}$ for visualizing the chloroplast. The second PMT (Trans detector) produces a bright-field version of the cell. The speed of the scanning was set to $200 \mathrm{~Hz}$, images were taken two seconds apart. In order to accurately compare the results of each test, these time-lapse images were quantified using ImageJ software and underwent statistical analysis using $\mathrm{R}$ software. The $30 \times 30 \mu \mathrm{m}$ illuminated section from the time series was contrast-enhanced with ImageJ (enhance contrast function), setting saturated pixels to $0 \%$. The mean fluorescence intensity was evaluated for all time points. For averaging across cells, the fluorescence values were normalized to the brightest time point in the series. For clearer data display, only 150 data points from the 600 available points were plotted for displayed. Plots were generated with $\mathrm{R}$ software.

\section{Results}

In the present work we investigate the participation of kinesin 4IIs in the chlo- 
roplast light avoidance response. We selected kinesin 4IIs because we had previously determined that they are present in $P$. patens but absent from $A$. thaliana [32]; this absence is similar to the microtubule-based chloroplast motility present in the moss $P$. patens, but absent in $A$. thaliana. We selected to use a loss-of-function strategy based on inducible RNAi [43]. This is a simple strategy because the protonemal cells can be subjected to estradiol induction by transferring them on cellophanes to plates with estradiol and waiting for the depletion of the kinesin 4 II.

\subsection{Phylogenetic Analysis Shows That Kinesin 4IIs Are Present in Algae, Bryophytes, and Lycopodiophyta, but Absent in Flowering Plants}

We have previously reported that in $P$. patens exists a subfamily of kinesin 4 proteins (kinesin 4IIs) that were not present in $A$. thaliana [32]. With the recent availability of several more genome sequences, here we have expanded our phylogenetic analysis to explore additional species. For this analysis we selected all algal species with a fully sequenced genome, all fully sequenced bryophytes (two mosses and one liverwort), the club moss Selaginella moellendorffii, and three representative angiosperms with the best-annotated genomes, pine apple, rice and $A$. thaliana. (For details see Materials and Methods section). After amino acid sequence alignment of their motor domains, we constructed a phylogenetic tree using the Maximum-Likelihood algorithm PhyML [44]. The tree topology is consistent with our previous analysis: in the green lineage, there are two clearly distinct subfamilies of kinesin 4s (Figure 1). The kinesin 4I subfamily has representatives from all the species analyzed, except for two algal species, Dunaliella salina and Ostreococcus lucimarinus, which do not have any kinesin 4 sequences of any type. The absence of kinesin 4 sequences in these two species is puzzling, but it may be the result of incomplete genome annotation; future analysis may help clarify this point. In contrast, kinesin II representatives are only present in some of the green algae species, in the bryophytes, and in $S$. moellendorffii. Algae and the liverwort $M$. polymorpha each has only one representative of each subfamily, while both mosses have multiple representatives for each. The club moss, $S$. moellendorffii, has multiple kinesin 4 I molecules, but only one kinesin 4II representative. Kinesin 4IIs from bryophytes and $S$. moellendorffii form a clear separate group, while the algae kinesin 4IIs form a less tight group. Kinesin 4Is have been considerably expanded in land plants, with bryophytes kinesin 4Is clustering separately from their angiosperm counterparts. Interestingly, the kinesin 4 Is from $S$. moellendorffii are distributed between the two groups, with two molecules clustering with each group, and a fifth molecule showing more divergence.

\subsection{Reduction of Kinesin 4-IIb Results in a Loss of Growth Phenotype}

We evaluated several RNAi lines designed to silence the kinesin 4II subfamily 


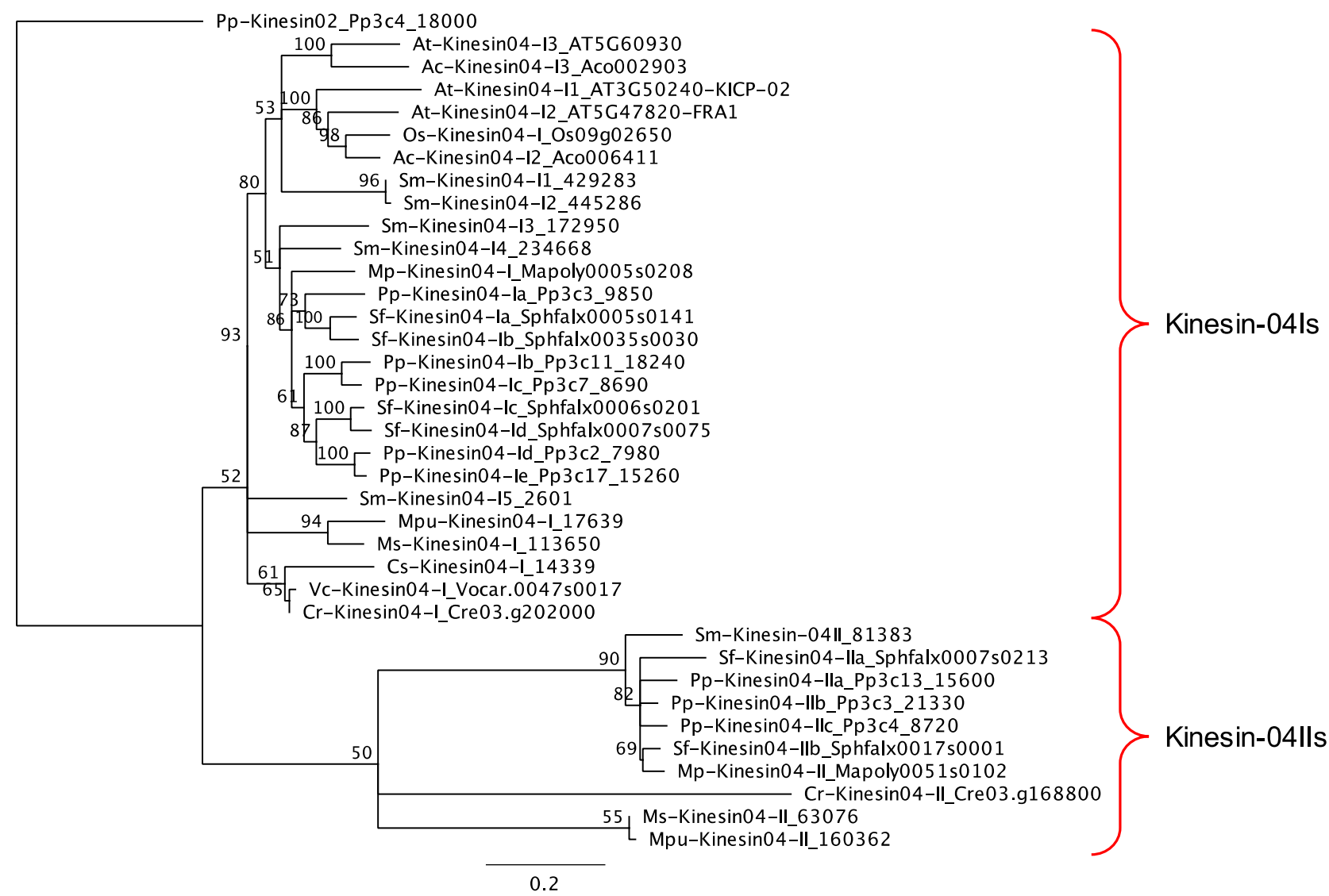

Figure 1. Phylogenetic analysis of selected members from plant and algal kinesin 4 family. The phylogenetic tree was constructed with the amino acid sequences corresponding to the kinesin's motor domain. Sequence alignment was done with ClustalW and the tree constructed with PhyML using Geneoius R7 sofwared. Percent bootstrap support above 50\% is indicated for each branch. Species names are as follows: Chlamydomonas reinhardtii (Cr), Volvox carteri (Vc), Coccomyxa subellipsoidea (Cs), Micromonas pusilla (Mpu), Micromonas sp. (Ms), Marchantia polymorpha (Mp), Physcomitrella patens (Pp), Sphagnum fallax (Sf), Selaginella moellendorffii (Sm), Ananas comosus (Ac), Oryza sativa (Os), and Arabidopsis thaliana (At). Motor domain was defined from alignment with the motor domain from the human kinesin 1 (see Materials and Methods and Supplementary Table 1 for details).

and identified one line that showed a consistent reduced-growth phenotype and our preliminary analysis suggested also an effect on chloroplast motility. In addition to the reduced growth that resulted when the kinesin 4II-RNAi line was placed on estradiol we observed less branching. We also observed a reduction in caulonemata differentiation and an abundance of shorter chloronemata (Figure 2, compare Figure 2(a) and Figure 2(b)). Another interesting feature of these abnormal growing plants was the fact that tip cells were slightly swollen (Figure 2(b)). This phenotype was reminiscent of cells where the polarization machinery has been disrupted [47] [48], suggesting a possible participation of kinesin 4IIs in cell polarization.

In order to quantify if the lack of kinesin 4II results in a significant growth phenotype, a growth assay was conducted. For this growth assay we used the RNAi-kinesin 4II line treated with estradiol and not treated, as well as the parental line originally used to generate the RNAi line, which was also treated with estradiol. The parental line acted as a control to confirm that the RNAi lines 


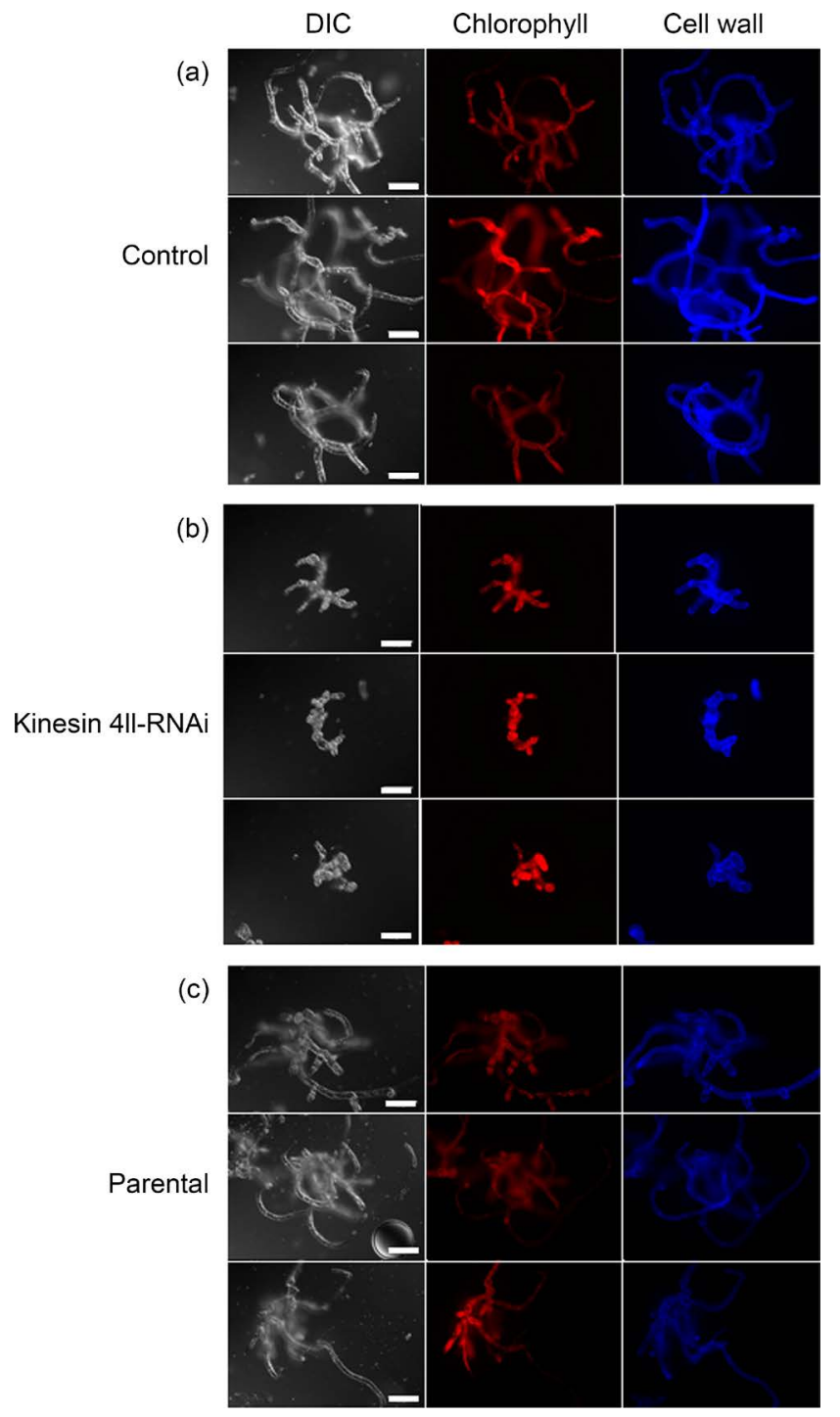

Figure 2. Silencing kinesin 4II results in a reduction in cell growth. (a) Control: single moss protoplasts from an inducible kinesin 4II-RNAi line were regenerated and not induced. (b) Kinesin 4II-RNAi: protoplasts from an inducible kinesin 4II-RNAi line were regenerated and RNAi induced with estradiol. (c) Parental control: single moss protoplasts from the parental cell line used to generate the kinesin 4II-RNAi line were regenerated and treated with estradiol. Images correspond to DIC (left panels), chlorophyll auto-fluorescence (middle panels), and cell wall staining by calcofluor (right panels). Scale bar $100 \mu \mathrm{m}$.

were not being affected by the estradiol treatment. Representative images for each of the conditions illustrate the consistency of the observed phenotype (Figure 2). Quantification of the morphological parameters, area and solidity, is shown in Figure 3. Both total area and solidity were significantly affected in the kinesin 4II-RNAi line (Figure 3(a) and Figure 3(b)). The significant reduction in area indicates that the plants are smaller, which is likely a result of a reduction in cellular growth, but cell numbers may also be affected. Solidity is the ratio between the plant's area and the convex hull's area, as this value increases the plants have a more "solid" morphology, which can be interpreted as a reduction 
(a)
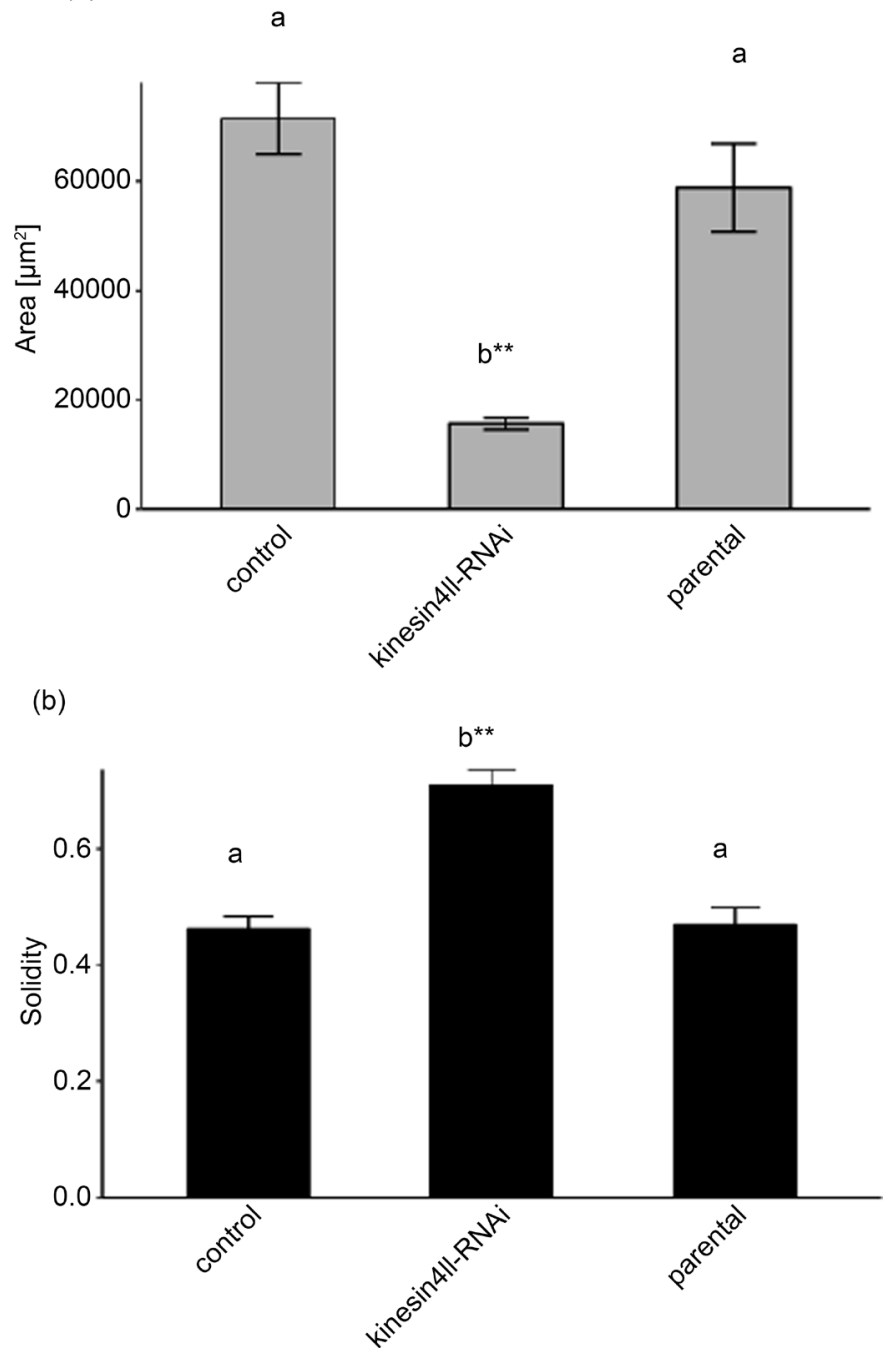

Figure 3. Statistical analysis of the morphological parameters shows that kinesin $4 \mathrm{II}$ is important for protonemal growth. (a) Comparison of the average values of area across different treatments and lines. Parental lines treated with estradiol have no significant change to their area. The control shows that estradiol has no direct affect to plant growth. (b) Comparison of the average values of solidity across different treatments and lines. Control values are very similar to parental lines that are treated with estradiol. Number of plants analyzed: 21 for control, 21 for kinesin $4 \mathrm{II}-\mathrm{RNAi}$, and 18 for the parental line. Low case letters indicate statistical groups (ANOVA-Tukey) with asterisks denoting an adjusted $\mathrm{P}<0.001$. Errors indicate SEM.

of cell polarization and branching. The observed significant increase in solidity in kinesin 4II-RNAi plants is consistent with the smaller plants having a reduction in polarization and branching, further supporting the participation of kinesin 4IIs in some aspect of cellular expansion. It is important to emphasize that we did not detect a difference in the overall area and solidity values between the control (untreated) line and the parental line treated with estradiol (Figure 2 and Figure 3). This indicates that any growth differences observed were a direct result of the induced RNAi lines and not the presence of estradiol. 


\subsection{Kinesin 4IIs Are Important for the Chloroplast Light Avoidance Response}

Because of the observed growth phenotype in the kinesin 4II-RNAi line, which resulted in small cells, it was necessary to establish conditions to visualize chloroplast motility in well-developed cells. This was provided by the versatility of the inducible RNAi system. To allow for the cells to expand before performing the chloroplast motility assay, the kinesin 4II-RNAi plants were first cultured for a full week in regular medium and subsequently transferred to estradiol plates for three days; this resulted in a ten-day culture process before imaging. The control line was just plated on $\mathrm{PpNO}_{3}$ plates for ten days. Following this procedure, fully elongated cells were available where the kinesin 4II RNAi system was induced.

Fully elongated sub-apical cells were selected that morphologically matched the untreated cells, and time series were collected to evaluate the chloroplast light avoidance response. Because in $P$. patens the chloroplast avoidance response can be mediated by either the actin or the microtubule cytoskeleton [8], we depolymerized the actin cytoskeleton to focus on microtubule-dependent processes and avoid confusion while interpreting our data. To depolymerize the actin cytoskeleton, we used the actin monomer-sequestering small molecule, latrunculin B, at a concentration known to disrupt the actin cytoskeleton [45]. During the assay the cells were exposed to blue light at their central region and chloroplast motility followed by imaging chloroplast using chlorophyll fluorescence and brightfield imaging. Images were acquired at 2 sec. intervals, which due to the required confocal laser scanning acquisition time results in an almost continuous light exposure. This light exposure robustly stimulates the light avoidance response, which is evident just a few minutes after exposure (Figure $4(a))$. In control cells, chloroplast moved almost completely out of the highly irradiated zone after $20 \mathrm{~min}$. of constant illumination (Figure 4(a)). In contrast, in the induced kinesin 4II-RNAi line, the chloroplasts do not seem to move to the same extent after $20 \mathrm{~min}$. of constant illumination (Figure 4(b)). However, looking at the time series (Supplementary Movies 1 and 2) and by quantifying the data (see below) some movement of the chloroplasts can still be detected.

To compare the magnitude and rate of the chloroplast light avoidance response between the control and induced kinesin 4II-RNAi lines, the fluorescence signal decay in the illuminated zone was measured and plotted as a function of time (Figure 5). As the chloroplast move out of the irradiated zone, the mean value for the chloroplast fluorescence signal is reduced. To correct for bleaching we normalized the analyzed images, using a linear histogram stretching, previous to quantifying the fluorescence. But, as is evident from the bright field images, the chloroplasts clearly move out of the irradiated region in control cells, and the light avoidance response is the main factor generating the reduction in fluorescent signal. Finally, to be able to compare across cells, the mean value of the fluorescence signal from each time point was divided by the mean value of the brightest time point of the series. The dynamics of chloroplast blue light 

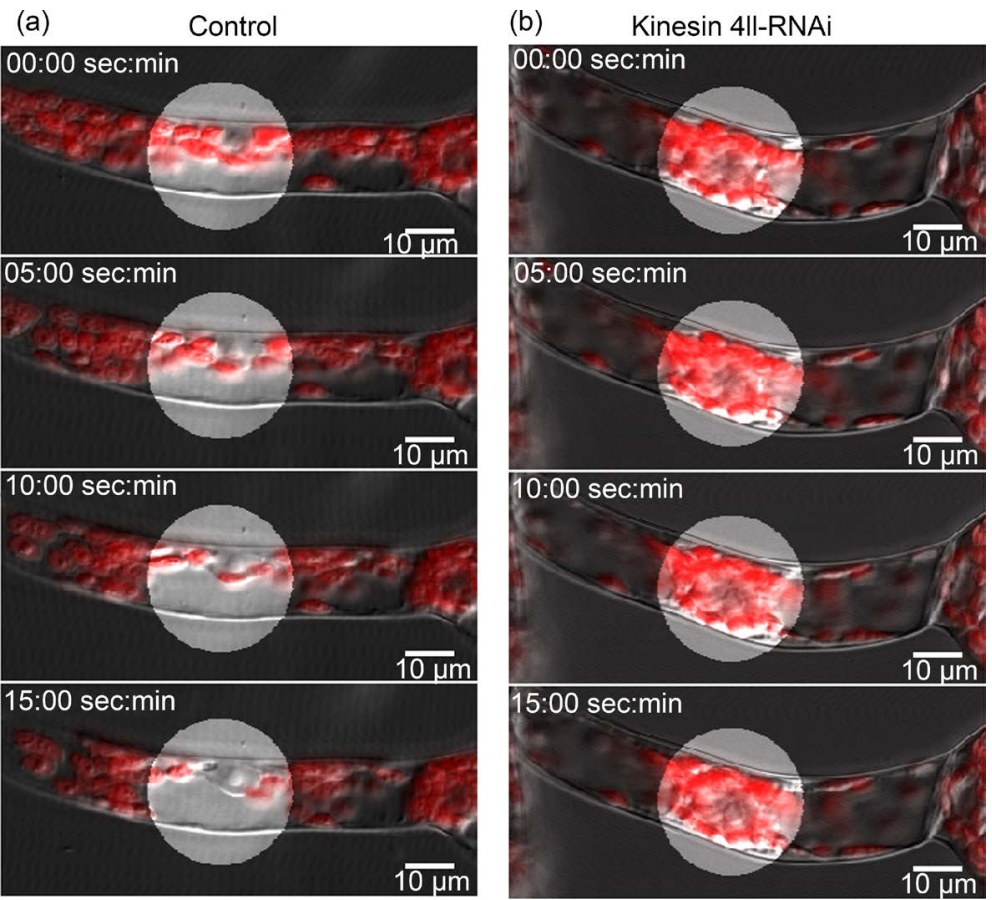

Figure 4. Kinesin 4II is necessary for the chloroplast light-avoidance response. Protonemal cells were irradiated within small area near the center to induce a blue light avoidance response from the chloroplasts. DIC (grey) and chlorophyll auto-fluorescence (red) images are superimposed. (a) Control cell where the chloroplasts are seen leaving the irradiated area. (b) Kinesin 4II-RNAi cell induced with estradiol. Cell shows some avoidance signal but most chloroplasts stay in the irradiated area.

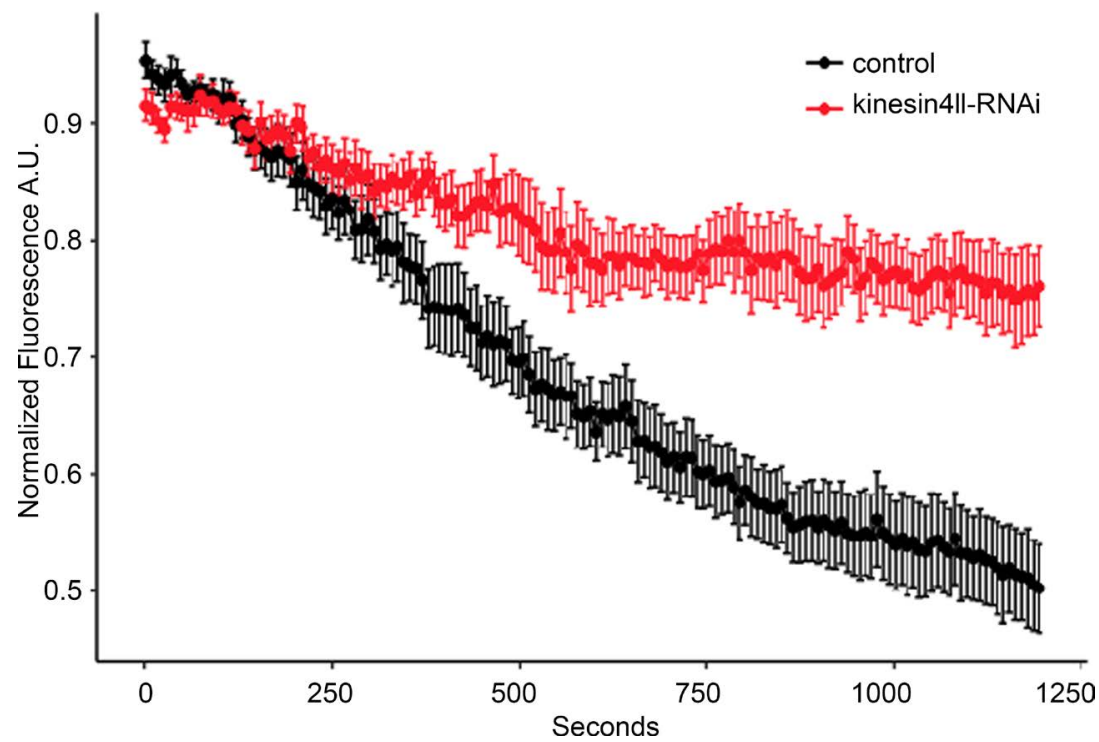

Figure 5. Quantification of chloroplast dynamics during the light avoidance response shows a reduction of chloroplast motility in kinesin 4-II-RNAi plants. Chloroplast avoidance was measured by tracking chlorophyll auto-fluorescence in the illuminated area. For each cell, the mean fluorescent signal for each time point was divided (normalized) by the signal from the brightest time point. Eleven cells were analyzed for the control condition and ten cells analyzed for the kinesin 4II-RNAi condition (estradiol induced). A t-test was conducted for the last two time points, showing a highly significant difference $(\mathrm{P}<0.001)$. 
avoidance of control cells follow an initial constant rate, which is slightly reduced after $10 \mathrm{~min}$., reaching a minimum value after $20 \mathrm{~min}$. (Figure 5). Kinesin 4II-RNAi cells also follow a constant avoidance rate during the initial $10 \mathrm{~min}$., but the rate is slower than that of control cells (Figure 5). After $10 \mathrm{~min}$. the kinesin 4II-RNAi cells seem to reach a plateau with very little light avoidance (Figure 5). Hence, it is clear that the chloroplasts still move in kinesin 4II-RNAi cells, but they do it at a much slower rate in response to blue light stimulation. To compare the statistical significance of the difference observed between control and kinesin 4II-RNAi cells, we compared the two last time points using statistical analysis and confirmed that the observed difference is highly significant (Figure 5).

\section{Discussion}

Here we showed that kinesin 4II proteins are important for cell growth in $P$. patens protonemata and that they participate in the chloroplast light-avoidance response. Kinesin 4IIs form a separate phylogenetic group, different from kine$\sin 4 \mathrm{Is}$, and are not present in flowering plants, hence they are good candidates for a role in chloroplast motility in $P$. patens. In this moss chloroplast transport can take place by an actin-dependent mechanism, conserved in vascular plants, and by a microtubule-dependent system, not present in vascular plants. Kinesin 4II may directly participate in chloroplast transport by binding to chloroplasts, or they may participate indirectly by modifying the microtubule cytoskeleton. Additional research will be necessary to determine which of these possibilities is correct. It is important to mention that we did not explore the participation of kinesin 4IIs in the chloroplast accumulation response, which in $P$. patens can also take place via actin-dependent and microtubule-dependent mechanisms. This is an important question that should be prioritized in future research; the availability of the inducible system used in this work should be valuable for this future work. It will also be interesting to explore the participation of kinesin 4IIs in the red-light dependent responses [6]. Because these are tubulin-dependent responses [8], it is likely kinesin 4IIs will play a role, but it is also possible that alternative kinesins are responsible for the red-light dependent system.

We did not anticipate identifying a reduced-growth phenotype in the kinesin 4II silenced plants, which suggests that, in addition to chloroplast motility, kinesin 4IIs participate in some aspect of growth. These growth effects may be specific for protonemata, but additional research is required to identify kinesin 4IIs participation in other aspects of moss development, such as gametophore development. The availability of an inducible system will provide a valuable system to explore other developmental stages.

In other systems, kinesins are motor proteins that have also been known to transport vesicles [49] and cell wall components [39] [40]. Because protonemata cell growth depends on secretory vesicle delivery [50], it is possible that the bulging of the tip cells observed in the kinesin 4II-RNAi plants results from a disruption of secretion, as has been observed in cells with a disrupted actin cy- 
toskeleton [47] [51]. The reduction on kinesin 4II levels could result in changes in regulation of vesicle fusion or in changes of the location of delivery, which is consistent with a cross-talk between the microtubule and actin cytoskeletons [52]. Further analysis will be needed to ascertain what cellular structures important for cell growth (vesicles, microtubules, etc.) are transported by kinesin 4II proteins.

The chloroplast motility assay presented here is equivalent to that performed by other groups [6] [8]. This is important, because for this work, a laser scanning confocal microscope was used, this system improves the reproducibility and simplicity of performing this assay, which is evident from looking at Figure 4 and Supplementary Movie 1. More importantly, the quantification clearly shows that the chloroplast light avoidance assay was highly reproducible and can be used to further explore effects on the chloroplast light avoidance response.

The hypothesis that kinesin 4II subfamily participates in chloroplasts transport is supported by the data presented here. Nevertheless, chloroplasts in the lines undergoing RNAi still moved to some degree after being illuminated with high intensity blue light. Some possible reasons that can explain why chloroplasts are only slowed and do not stop completely could include the following: First, it is likely that the inducible RNAi system does not deplete all the kinesin 4IIs present in the cytoplasm; hence there may still be some amount of kinesin $4 \mathrm{II}$ left in the cell, which could be moving the chloroplasts. Another possibility is that there may be a functionally redundant kinesin that is performing the function of kinesin 4II in chloroplast motility, but in a less efficient fashion. Finally, another possibility is that the lack of kinesin 4II may affect the orientation or dynamics of the microtubules in protonemata. To address this, it will be necessary to visualize the endogenous microtubules while inducing chloroplast photo-relocation. Although many questions remain about its function, the current work points to a critical role of kinesin 4IIs in $P$. patens development and adaptation to its environment.

\section{Acknowledgements}

We thank our colleagues from the Vidali and Tüzel Labs, notably Sakshi Khurana and Olivia Shraibati for their support. We also thank Dr. Gohta Goshima for providing the kinesin 4II-RNAi and parental lines. This project was supported by NSF grant MCB-1253444 to L.V.

\section{References}

[1] Menzel, D. and Schliwa, M. (1986) Motility in the Siphonous Green Alga Bryopsis. II. Chloroplast Movement Requires Organized Arrays of Both Microtubules and Actin Filaments. European Journal of Cell Biology, 40, 286-295.

[2] Davis, P.A. and Hangarter, R.P. (2012) Chloroplast Movement Provides Photoprotection to Plants by Redistributing PSII Damage within Leaves. Photosynthesis Research, 112, 153-161. https://doi.org/10.1007/s11120-012-9755-4

[3] Wada, M. (2013) Chloroplast Movement. Plant Science, 210, 177-182. https://doi.org/10.1016/j.plantsci.2013.05.016 
[4] Suetsugu, N. and Wada, M. (2009) Chloroplast Photorelocation Movement. In: Sandelius, A.S. and Aronsson, H., Eds., The Chloroplast. Interactions with the Environment, Vol. 13, Springer, Berlin Heidelberg, 235-266.

https://doi.org/10.1007/978-3-540-68696-5_8

[5] Kasahara, M., et al. (2002) Chloroplast Avoidance Movement Reduces Photodamage in Plants. Nature, 420, 829-832. https://doi.org/10.1038/nature01213

[6] Kadota, A., Sato, Y. and Wada, M. (2000) Intracellular Chloroplast Photorelocation in the Moss Physcomitrella patens Is Mediated by Phytochrome as Well as by a Blue-Light Receptor. Planta, 210, 932-937. https://doi.org/10.1007/s004250050700

[7] Kasahara, M., Kagawa, T., Sato, Y., Kiyosue, T. and Wada, M. (2004) Phototropins Mediate Blue and Red Light-Induced Chloroplast Movements in Physcomitrella patens. Plant Physiology, 135, 1388-1397. https://doi.org/10.1104/pp.104.042705

[8] Sato, Y., Wada, M. and Kadota, A. (2001) Choice of Tracks, Microtubules and/or Actin Filaments for Chloroplast Photo-Movement Is Differentially Controlled by Phytochrome and a Blue Light Receptor. Journal of Cell Science, 114, 269-279.

[9] Christie, J.M. (2007) Phototropin Blue-Light Receptors. Annual Review of Plant Biology, 58, 21-45. https://doi.org/10.1146/annurev.arplant.58.032806.103951

[10] DeBlasio, S.L., Mullen, J.L., Luesse, D.R. and Hangarter, R.P. (2003) Phytochrome Modulation of Blue Light-Induced Chloroplast Movements in Arabidopsis. Plant Physiology, 133, 1471-1479. https://doi.org/10.1104/pp.103.029116

[11] Kagawa, T., et al. (2001) Arabidopsis NPL1: A Phototropin Homolog Controlling the Chloroplast High-Light Avoidance Response. Science, 291, 2138-2141. https://doi.org/10.1126/science.291.5511.2138

[12] Kadota, A., et al. (2009) Short Actin-Based Mechanism for Light-Directed Chloroplast Movement in Arabidopsis. Proceedings of the National Academy of Sciences, 106, 13106-13111. https://doi.org/10.1073/pnas.0906250106

[13] Yamashita, H., et al. (2011) Chloroplast Actin Filaments Organize Meshwork on the Photorelocated Chloroplasts in the Moss Physcomitrella patens. Planta, 233, 357368. https://doi.org/10.1007/s00425-010-1299-2

[14] Shen, Z., et al. (2015) The Kinesin-Like Proteins, KAC1/2, Regulate Actin Dynamics Underlying Chloroplast Light-Avoidance in Physcomitrella patens. Journal of Integrative Plant Biology, 57, 106-119. https://doi.org/10.1111/jipb.12303

[15] Cove, D.J., et al. (2009) The Moss Physcomitrella patens. A Novel Model System for Plant Development and Genomic Studies. In: Cold Spring Harbor Laboratory Press, Emerging Model Organisms: A Laboratory Manual, Vol. 1, CSHL Press, Cold Spring Harbor. https://doi.org/10.1101/pdb.emo115

[16] Rensing, S.A., et al. (2008) The Physcomitrella Genome Reveals Evolutionary Insights into the Conquest of Land by Plants. Science, 319, 64-69.

https://doi.org/10.1126/science.1150646

[17] Jenkins, G.I. and Cove, D.J. (1983) Phototropism and Polarotropism of Primary Chloronemata of the Moss Physcomitrella patens-Responses of Mutant Strains. Planta, 159, 432-438. https://doi.org/10.1007/BF00392079

[18] Schaefer, D.G. and Zryd, J.P. (1997) Efficient Gene Targeting in the Moss Physcomitrella patens. Plant Journal, 11, 1195-1206. https://doi.org/10.1046/j.1365-313X.1997.11061195.x

[19] Kamisugi, Y., et al. (2006) The Mechanism of Gene Targeting in Physcomitrella patens. Homologous Recombination, Concatenation and Multiple Integration. Nucleic Acids Research, 34, 6205-6214. https://doi.org/10.1093/nar/gkl832

[20] Bezanilla, M., Pan, A. and Quatrano, R.S. (2003) RNA Interference in the Moss 
Physcomitrella patens. Plant Physiology, 133, 470-474.

https://doi.org/10.1104/pp.103.024901

[21] Nakaoka, Y., et al. (2012) An Inducible RNA Interference System in Physcomitrella patens Reveals a Dominant Role of Augmin in Phragmoplast Microtubule Generation. Plant Cell, 24, 1478-1493. https://doi.org/10.1105/tpc.112.098509

[22] Zurzycki, J. (1967) Properties and Localization of the Photoreceptor Active in Displacements of Chloroplasts in Funaria hygrometrica. I. Action Spectrum. Acta Societatis Botanicorum Poloniae, 36, 133-142. https://doi.org/10.5586/asbp.1967.012

[23] Kagawa, T., Lamparter, T., Hartmann, E. and Wada, M. (1997) Phytochrome-Mediated Branch Formation in Protonemata of the Moss Ceratodon purpureus. Journal of Plant Research, 110, 363-370. https://doi.org/10.1007/BF02524935

[24] Usami, H., et al. (2012) CHUP1 Mediates Actin-Based Light-Induced Chloroplast Avoidance Movement in the Moss Physcomitrella patens. Planta, 236, 1889-1897. https://doi.org/10.1007/s00425-012-1735-6

[25] Suetsugu, N., et al. (2012) The KAC Family of Kinesin-Like Proteins Is Essential for the Association of Chloroplasts with the Plasma Membrane in Land Plants. Plant and Cell Physiology, 53, 1854-1865. https://doi.org/10.1093/pcp/pcs133

[26] Suetsugu, N. and Wada, M. (2016) Evolution of the Cp-Actin-Based Motility System of Chloroplasts in Green Plants. Frontiers in Plant Science, 7, 561. https://doi.org/10.3389/fpls.2016.00561

[27] Richardson, D., Simmons, M. and Reddy, A. (2006) Comprehensive Comparative Analysis of Kinesins in Photosynthetic Eukaryotes. BMC Genomics, 7, 1-37. https://doi.org/10.1186/1471-2164-7-18

[28] Hirokawa, N., Noda, Y. and Okada, Y. (1998) Kinesin and Dynein Superfamily Proteins in Organelle Transport and Cell Division. Current Opinion in Cell Biology, 10, 60-73. https://doi.org/10.1016/S0955-0674(98)80087-2

[29] Hiwatashi, Y., et al. (2008) Kinesins Are Indispensable for Interdigitation of Phragmoplast Microtubules in the Moss Physcomitrella patens. Plant Cell, 20, 3094-3106. https://doi.org/10.1105/tpc.108.061705

[30] Lee, Y.R., Li, Y. and Liu, B. (2007) Two Arabidopsis Phragmoplast-Associated Kinesins Play a Critical Role in Cytokinesis During Male Gametogenesis. Plant Cell, 19, 2595-2605. https://doi.org/10.1105/tpc.107.050716

[31] Lee, Y.R. and Liu, B. (2000) Identification of a Phragmoplast-Associated Kinesin-Related Protein in Higher Plants. Current Biology, 10, 797-800. https://doi.org/10.1016/S0960-9822(00)00564-9

[32] Shen, Z., Collatos, A.R., Bibeau, J.P., Furt, F. and Vidali, L. (2012) Phylogenetic Analysis of the Kinesin Superfamily from Physcomitrella. Frontiers in Plant Science, 3, 230. https://doi.org/10.3389/fpls.2012.00230

[33] Miki, T., Naito, H., Nishina, M. and Goshima, G. (2014) Endogenous Localizome Identifies 43 Mitotic Kinesins in a Plant Cell. Proceedings of the National Academy of Sciences, 111, E1053-E1061. https://doi.org/10.1073/pnas.1311243111

[34] Suetsugu, N., et al. (2010) Two Kinesin-Like Proteins Mediate Actin-Based Chloroplast Movement in Arabidopsis thaliana. Proceedings of the National Academy of Sciences, 107, 8860-8865. https://doi.org/10.1073/pnas.0912773107

[35] Hirokawa, N., Noda, Y., Tanaka, Y. and Niwa, S. (2009) Kinesin Superfamily Motor Proteins and Intracellular Transport. Nature Reviews Molecular Cell Biology, 10, 682-696. https://doi.org/10.1038/nrm2774

[36] Zhong, R., Burk, D.H., Morrison, W.H. and Ye, Z.H. (2002) A Kinesin-Like Protein Is Essential for Oriented Deposition of Cellulose Microfibrils and Cell Wall 
Strength. Plant Cell, 14, 3101-3117. https://doi.org/10.1105/tpc.005801

[37] Zhang, M., et al. (2010) Brittle Culm 12, a Dual-Targeting Kinesin-4 Protein, Controls Cell-Cycle Progression and Wall Properties in Rice. The Plant Journal, 63, 312-328. https://doi.org/10.1111/j.1365-313X.2010.04238.x

[38] Zhu, C. and Dixit, R. (2011) Single Molecule Analysis of the Arabidopsis FRA1 Kinesin Shows That It Is a Functional Motor Protein with Unusually High Processivity. Molecular Plant, 4, 879-885. https://doi.org/10.1093/mp/ssr077

[39] Zhu, C., et al. (2015) The Fragile Fiber1 Kinesin Contributes to Cortical Microtubule-Mediated Trafficking of Cell Wall Components. Plant Physiology, 167, 780 792. https://doi.org/10.1104/pp.114.251462

[40] Kong, Z., et al. (2015) Kinesin-4 Functions in Vesicular Transport on Cortical Microtubules and Regulates Cell Wall Mechanics during Cell Elongation in Plants. Molecular Plant, 8, 1011-1023. https://doi.org/10.1016/j.molp.2015.01.004

[41] Hu, C.K., Coughlin, M., Field, C.M. and Mitchison, T.J. (2011) Kif4 Regulates Midzone Length During Cytokinesis. Current Biology, 21, 815-824.

https://doi.org/10.1016/j.cub.2011.04.019

[42] Kubo, M., et al. (2013) System for Stable Beta-Estradiol-Inducible Gene Expression in the Moss Physcomitrella patens. PLoS ONE, 8, e77356. https://doi.org/10.1371/journal.pone.0077356

[43] Miki, T., Nishina, M. and Goshima, G. (2015) RNAi Screening Identifies the Armadillo Repeat-Containing Kinesins Responsible for Microtubule-Dependent Nuclear Positioning in Physcomitrella patens. Plant and Cell Physiology, 56, 737-749. https://doi.org/10.1093/pcp/pcv002

[44] Guindon, S. and Gascuel, O. (2003) A Simple, Fast and Accurate Algorithm to Estimate Large Phylogenies by Maximum Likelihood. Systematic Biology, 52, 696-704. https://doi.org/10.1080/10635150390235520

[45] Vidali, L., Rounds, C.M., Hepler, P.K. and Bezanilla, M. (2009) Lifeact-mEGFP Reveals a Dynamic Apical F-Actin Network in Tip Growing Plant Cells. PLoS ONE, 4, e5744. https://doi.org/10.1371/journal.pone.0005744

[46] Bibeau, J.P. and Vidali, L. (2014) Morphological Analysis of Cell Growth Mutants in Physcomitrella. Methods in Molecular Biology, 1080, 201-213. https://doi.org/10.1007/978-1-62703-643-6_17

[47] Vidali, L., Augustine, R.C., Kleinman, K.P. and Bezanilla, M. (2007) Profilin Is Essential for Tip Growth in the Moss Physcomitrella patens. Plant Cell, 19, 3705-3722. https://doi.org/10.1105/tpc.107.053413

[48] Vidali, L., et al. (2010) Myosin XI Is Essential for Tip Growth in Physcomitrella patens. Plant Cell, 22, 1868-1882. https://doi.org/10.1105/tpc.109.073288

[49] Vale, R.D. (2003) The Molecular Motor Toolbox for Intracellular Transport. Cell, 112, 467-480. https://doi.org/10.1016/S0092-8674(03)00111-9

[50] Furt, F., Liu, Y.C., Bibeau, J.P., Tüzel, E. and Vidali, L. (2013) Apical Myosin XI Anticipates F-Actin during Polarized Growth of Physcomitrella patens Cells. The Plant Journal, 73, 417-428. https://doi.org/10.1111/tpj.12039

[51] Vidali, L., et al. (2009) Rapid Formin-Mediated Actin-Filament Elongation Is Essential for Polarized Plant Cell Growth. Proceedings of the National Academy of Sciences, 106, 13341-13346. https://doi.org/10.1073/pnas.0901170106

[52] Wu, S.Z. and Bezanilla, M. (2014) Myosin VIII Associates with Microtubule Ends and Together with Actin Plays a Role in Guiding Plant Cell Division. eLife, 3, e03498. https://doi.org/10.7554/elife.03498 


\section{Appendix}

Supplementary Table 1. Protein sequences used for the phylogenetic analysis of the plant kinesin 4 family.

\begin{tabular}{|c|c|c|}
\hline Phylogenetic tree name ${ }^{a}$ & Peptide name in Phytozome & $\begin{array}{c}\text { Motor } \\
\text { domain }^{\mathrm{b}}\end{array}$ \\
\hline Cr-Kinesin04-II_Cre03.g168800 & Org_Creinhardtii peptide: Cre03.g168800.t1 & $14-404$ \\
\hline Cr-Kinesin04-I_Cre03.g202000 & Org_Creinhardtii peptide: Cre03.g202000.t1 & $18-377$ \\
\hline Vc-Kinesin04-I_Vocar.0047s0017 & Org_Vcarteri peptide: Vocar.0047s0017.1 & $13-364$ \\
\hline Cs-Kinesin04-I_14339 & Org_CsubellipsoideaC-169 peptide: 14339 & $18-366$ \\
\hline Mpu-Kinesin04-I_17639 & Org_MpusillaCCMP1545 peptide: 17639 & $18-351$ \\
\hline Mpu-Kinesin04-II_160362 & Org_MpusillaCCMP1545 peptide: 160362 & $81-429$ \\
\hline Ms-Kinesin04-I_113650 & Org_Msp.RCC299 peptide: 113650 & $31-363$ \\
\hline Ms-Ms-Kinesin04-II_63076 & Org_Msp.RCC299 peptide: 63076 & $77-418$ \\
\hline Mp-Kinesin04-I_Mapoly0005s0208 & $\begin{array}{l}\text { Org_Mpolymorpha peptide: } \\
\text { Mapoly0005s } 0208.1\end{array}$ & 23-391 \\
\hline Mp-Kinesin04-II_Mapoly0051s0102 & $\begin{array}{l}\text { Org_Mpolymorpha peptide: } \\
\text { Mapoly0051s0102.1 }\end{array}$ & 63-394 \\
\hline Pp-Kinesin04-Ia_Pp3c3_9850 & Org_Ppatens peptide: Pp3c3_9850V3.1 & $1-366$ \\
\hline Pp-Kinesin04-Ib_Pp3c11_18240 & Org_Ppatens peptide: Pp3c11_18240V3.1 & $31-393$ \\
\hline Pp-Kinesin04-Ic_Pp3c7_8690 & Org_Ppatens peptide: Pp3c7_8690V3.1 & 29-386 \\
\hline Pp-Kinesin04-Id_Pp3c2_7980 & Org_Ppatens peptide: Pp3c2_7980V3.1 & $26-383$ \\
\hline Pp-Kinesin04-Ie_Pp3c17_15260 & Org_Ppatens peptide: Pp3c17_15260V3.1 & $28-385$ \\
\hline Pp-Kinesin04-IIa_Pp3c13_15600 & Org_Ppatens peptide: Pp3c13_15600V3.1 & $21-352$ \\
\hline Pp-Kinesin04-IIb_Pp3c3_21330 & Org_Ppatens peptide: Pp3c3_21330V3.1 & $21-330$ \\
\hline Pp-Kinesin04-IIc_Pp3c4_8720 & Org_Ppatens peptide: Pp3c4_8720V3.1 & $21-351$ \\
\hline Sf-Kinesin04-Ia_Sphfalx0005s0141 & Org_Sfallax peptide: Sphfalx0005s0141.1 & $32-298$ \\
\hline Pp-Kinesin02_Pp3c4_18000 & Org_Ppatens peptide: Pp3c4_18000V3.1 & $34-371$ \\
\hline Sf-Kinesin04-Ib_Sphfalx0035s0030 & Org_Sfallax peptide: Sphfalx0035s0030.1 & $12-387$ \\
\hline Sf-Kinesin04-Ic_Sphfalx0006s0201 & Org_Sfallax peptide: Sphfalx0006s0201.1 & $31-389$ \\
\hline Sf-Kinesin04-Id_Sphfalx0007s0075 & Org_Sfallax peptide: Sphfalx0007s0075.1 & $30-388$ \\
\hline Sf-Kinesin04-IIa_Sphfalx0007s0213 & Org_Sfallax peptide: Sphfalx0007s0213.1 & $55-390$ \\
\hline Sf-Kinesin04-IIb_Sphfalx0017s0001 & Org_Sfallax peptide: Sphfalx0017s0001.1 & $30-361$ \\
\hline Sm-Kinesin04-I1_429283 & Org_Smoellendorffii peptide: 429283 & $14-366$ \\
\hline Sm-Kinesin04-I2_445286 & Org_Smoellendorffii peptide: 445286 & $14-367$ \\
\hline Sm-Kinesin04-I3_172950 & Org_Smoellendorffii peptide: 172950 & $27-379$ \\
\hline Sm-Kinesin04-I4_234668 & Org_Smoellendorffii peptide: 234668 & $1-272$ \\
\hline Sm-Kinesin04-I5_2601 & Org_Smoellendorffii peptide: 2601 & $1-290$ \\
\hline Sm-Kinesin-04II_81383 & Org_Smoellendorffii peptide: 81383 & $21-361$ \\
\hline Ac-Kinesin04-I2_Aco006411 & Org_Acomosus peptide: Aco006411.1 & $10-368$ \\
\hline Ac-Kinesin04-I3_Aco002903 & Org_Acomosus peptide: Aco002903.1 & $14-357$ \\
\hline Os-Kinesin04-I_Os09g02650 & Org_Osativa peptide: LOC_Os09g02650.1 & $10-369$ \\
\hline At-Kinesin04-I1_AT3G50240-FRA1 & Org_Athaliana peptide: AT3G50240.1 & $25-380$ \\
\hline At-Kinesin04-I2_AT5G47820 & Org_Athaliana peptide: AT5G47820.1 & $11-370$ \\
\hline At-Kinesin04-I3_AT5G60930 & Org_Athaliana peptide: AT5G60930.1 & $6-360$ \\
\hline
\end{tabular}

${ }^{a}$ The species names and their abbreviations used here and in Figure 1 are as follows: Chlamydomonas reinhardtii (Cr), Volvox carteri (Vc), Coccomyxa subellipsoidea (Cs), Micromonas pusilla (Mpu), Micromonas sp. (Ms), Marchantia polymorpha (Mp), Physcomitrella patens (Pp), Sphagnum fallax (Sf), Selaginella moellendorffii (Sm), Ananas comosus (Ac), Oryza sativa (Os) and Arabidopsis thaliana (At); ${ }^{\mathrm{b}} \mathrm{Motor}$ domain was defined from alignment with the motor domain from the human kinesin 1, amino acids selected from the protein derived from the gene model in Phytozome are indicated. 


\section{Supplementary Movie Legends (see http://ajps2017.vidalilab.net/)}

Supplementary Movie 1. Control cell where the chloroplasts are seen leaving the irradiated area. Protonemal cells were irradiated within small area near the center to induce a blue light avoidance response from the chloroplasts. DIC (grey) and chlorophyll auto-fluorescence (red) images are superimposed.

Supplementary Movie 2. Kinesin 4II-RNAi cell induced with estradiol. Cell shows some avoidance signal but most chloroplasts stay in the irradiated area. Protonemal cells were irradiated within small area near the center to induce a blue light avoidance response from the chloroplasts. DIC (grey) and chlorophyll auto-fluorescence (red) images are superimposed.

\section{Scientific Research Publishing}

Submit or recommend next manuscript to SCIRP and we will provide best service for you:

Accepting pre-submission inquiries through Email, Facebook, LinkedIn, Twitter, etc. A wide selection of journals (inclusive of 9 subjects, more than 200 journals)

Providing 24-hour high-quality service

User-friendly online submission system

Fair and swift peer-review system

Efficient typesetting and proofreading procedure

Display of the result of downloads and visits, as well as the number of cited articles Maximum dissemination of your research work

Submit your manuscript at: http://papersubmission.scirp.org/

Or contact ajps@scirp.org 